\title{
BMJ Open Needs assessment for a decision support tool in oral cancer requiring major resection and reconstruction: a mixed- methods study protocol
}

\author{
David Forner (D , , ${ }^{1,2}$ Paul Hong, ${ }^{1,3}$ Martin Corsten, ${ }^{1}$ Valeria E Rac, ${ }^{2,4}$ \\ Rosemary Martino, ${ }^{5}$ Andrew G Shuman, ${ }^{6}$ Douglas B Chepeha, ${ }^{7}$ Anna M Sawka, ${ }^{8}$ \\ John R de Almeida, ${ }^{2,7}$ Jonathan C Irish, ${ }^{7}$ Dale H Brown, ${ }^{7}$ S Mark Taylor, ${ }^{1}$ \\ Patrick J Gullane, ${ }^{7}$ Jonathan R Trites, ${ }^{1}$ Ralph Gilbert, ${ }^{7}$ Matthew H Rigby, ${ }^{1}$ \\ Jolie Ringash, ${ }^{2,9}$ David Goldstein ${ }^{7}$
}

To cite: Forner $D$, Hong $P$, Corsten $\mathrm{M}$, et al. Needs assessment for a decision support tool in oral cancer requiring major resection and reconstruction: $\mathrm{a}$ mixed-methods study protocol. BMJ Open 2020;10:e036969. doi:10.1136/ bmjopen-2020-036969

- Prepublication history and additional material for this paper are available online. To view these files, please visit the journal online (http://dx.doi org/10.1136/bmjopen-2020036969).

Received 13 January 2020 Revised 22 August 2020 Accepted 07 October 2020
Check for updates

(C) Author(s) (or their employer(s)) 2020. Re-use permitted under CC BY-NC. No commercial re-use. See rights and permissions. Published by BMJ.

For numbered affiliations see end of article.

Correspondence to

Dr David Goldstein;

David.Goldstein@uhn.ca

\section{ABSTRACT}

Introduction Advanced oral cancer and its ensuing treatment engenders significant morbidity and mortality. Patients are often elderly with significant comorbidities. Toxicities associated with surgical resection can be devastating and they are often highlighted by patients as impactful. Given the potential for suboptimal oncological and functional outcomes in this vulnerable patient population, promotion and performance of shared decision making (SDM) is crucial.

Decision aids (DAs) are useful instruments for facilitating the SDM process by presenting patients with up-todate evidence regarding risks, benefits and the possible postoperative course. Importantly, DAs also help elicit and clarify patient values and preferences. The use of DAs in cancer treatment has been shown to reduce decisional conflict and increase SDM. No DAs for oral cavity cancer have yet been developed.

This study endeavours to answer the question: Is there a patient or surgeon driven need for development and implementation of a DA for adult patients considering major surgery for oral cancer?

Methods and analysis This study is the first step in a multiphase investigation of SDM during major head and neck surgery. It is a multi-institutional convergent parallel mixed-methods needs assessment study. Patients and surgeon dyads will be recruited to complete questionnaires related to their perception of the SDM process (nine-item Shared Decision-Making Questionnaire, SDM-Q-9 and SDM-Q-Doc) and to take part in semistructured interviews. Patients will also complete questionnaires examining decisional self-efficacy (0ttawa Decision Self-Efficacy Scale) and decisional conflict (Decisional Conflict Scale). Questionnaires will be completed at time of recruitment and will be used to assess the current level of SDM, selfefficacy and conflict in this setting. Thematic analysis will be used to analyse transcripts of interviews. Quantitative and qualitative components of the study will be integrated through triangulation, with matrix developed to promote visualisation of the data.

Ethics and Dissemination This study has been approved by the research ethics boards of the Nova Scotia Health

\section{Strengths and limitations of this study}

- This study represents the first formal needs assessment for a decision aid in oral cancer.

- The mixed-methods approach used in this study will allow an in-depth understanding of patient and surgeon perceptions of the current shared decisionmaking landscape.

- Although the use of individual semistructured interviews is time consuming and elevates the total study time and cost, the use of this methodology is essential to promote sharing of personal and emotional information given the sensitive nature of a cancer diagnosis.

- This study does not directly assess how the values and preferences of caregivers, family, friends and other individuals involved in the life of participants. Such individuals may offer important considerations.

- Patients experiencing substantial distress may be those who most benefit from a decision aid, but may also not wish to participate in the study, introducing an inherent bias in the study.

Authority (Halifax, Nova Scotia) and the University Health Network (Toronto, Ontario). Dissemination to clinicians will be through traditional approaches and creation of a head and neck cancer SDM website. Dissemination to patients will include a section within the website, patient advocacy groups and postings within clinical environments.

\section{INTRODUCTION}

Surgical resection and reconstruction is the primary treatment for advanced oral cancer. Treatment-associated complications and long-term disability, particularly related to speech, swallowing and cosmesis, can be substantial. Difficulty with eating and speech are often highlighted by patients as functionally and socially impactful. ${ }^{1}$ In addition, many patients with head and neck cancer are 
older and frequently have comorbidities related to age and cancer-associated risk factors. ${ }^{2}$ Furthermore, due to both cancer cachexia and the tumour effect on the upper aerodigestive tract, patients are often severely malnourished on presentation. ${ }^{3}$

Given the potential for poor oncological and functional outcomes in this vulnerable patient population, patient-centred care through shared decision making is crucial. There is a growing body of evidence that unmet information needs may contribute significantly to psychological distress. ${ }^{4}$ Difficult decisions surrounding cancer and its treatment place patients in a vulnerable position that is worsened when their understanding of management options and post-treatment expectations is lacking. For example, patients may be uncertain how their speech and swallowing ability may be affected following reconstruction with free tissue transfer, making the decision to undergo treatment even more difficult. However, this area is understudied in the head and neck cancer population and there is a paucity of evidence regarding patient and surgeon experiences. As patients may face drastically different issues from the immediate to late post-treatment periods, accurate communication of expectations is key.

Shared decision making is a collaborative process that allows patients and their healthcare providers to make medical decisions together, using the scientific expertise of the healthcare team, while considering the values and preferences of the patient themselves. Patient perceptions of their involvement in the shared decision-making process is associated with improved affective cognitive and health outcomes. ${ }^{5}$

Owing to the burdens of both the disease and its treatment, the shared decision-making process is particularly important to determine how patients grapple with the consideration of undergoing surgery for head and neck cancer. Previous studies have shown that many patients experience significant decisional regret after treatment, and their degree of regret is dependent on specific treatment modalities. ${ }^{67}$ Thomas et alrecently reported that $27 \%$ of patients with head and neck cancer 50 years and older undergoing major head and neck surgery had moderate to severe regret scores following surgery. ${ }^{8}$ Shuman et al have shown that patients who value swallowing above all other functions in the preoperative period report less regret in the postoperative period when their swallowing outcome is satisfactory-the same observation holds true for voice related outcomes. ${ }^{9}$ While surgery is the primary treatment modality for advanced oral cancer, significant permanent alterations in patient speech and swallowing can lead to substantial disability and reduced quality of life. In older patients with multiple comorbidities, cure rates may be relatively low, and morbidity may be relatively high. Under such circumstances, patients may wish to pursue non-surgical options such as palliative and supportive care. Patients must be fully informed of all potential options, as treatment goals can be exceptionally individualised. This further highlights the importance of elucidating patient values and preferences in the pretreatment period. In the context of head and neck cancer treatment, patients may regret having undergone invasive treatment if they ultimately experience substantial morbidity with limited survival benefit.

Decision aids are decision support tools for improving the shared decision-making process by presenting patients with evidence regarding risks, benefits and the possible postoperative outcomes for different management options. The use of decision aids for cancer treatment has been shown to be useful in reducing decisional conflict, bolstering knowledge, improving value-choice congruence and increasing involvement in the shared decision-making process. ${ }^{10}$ The goal of implementing a decision aid is not to replace the personal interaction between patients and their providers, but instead to help facilitate shared decision making. To-date, no decision aids for oral cavity cancer have yet been developed.

This study is a needs assessment to determine if patients considering major surgery for oral cavity cancer believe they would benefit from assistance in treatment decision making. While patients weigh their consideration to undergo major surgery against supportive care and palliative treatment options, the focus of this study is on the patient-surgeon dyad and the integration of surgical expectations with patient values and preferences. Thus, the decision to be considered by patients and their healthcare team is whether to undergo standard of care treatment (major surgery) or non-standard of care treatment, including supportive care or palliative management options. This needs assessment is the first phase in an overarching multiphase study examining the shared decision-making process in major head and neck cancer. Needs assessments are crucial to perform before embarking on the development of a decision aid as their development can be resource intensive and may require multiple systematic literature searches, qualitative interviews, the involvement of many participants and several rounds of development, assessment and revision. Multiple stakeholders must be engaged during the multidisciplinary development process, including patients, physicians, allied health professionals and decision aid development experts.

We, therefore, aim to answer the question: Is there a patient or surgeon driven need for development and implementation of a decision aid for adult patients recommended to have major surgery for oral cavity cancer? For the purpose of this study, 'major surgery' is considered to be that for which regional or free flap reconstruction is required. This study advances knowledge about the decision-making process of major oral cancer surgery, including providing surgeons, patients, policy makers, researchers and other key stakeholders with knowledge about how to optimise patient-centred care in this setting.

\section{METHODS AND ANALYSIS}

This protocol was prepared in accordance with elements of the Strengthening the Reporting of Observational 


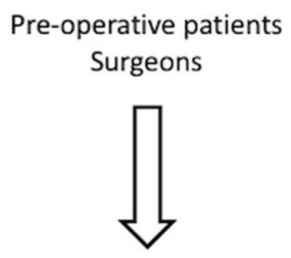

Questionnaires

(Quantitative)

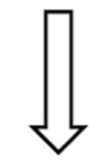

Interviews

(Qualitative)

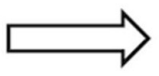

Post-operative patients Surgeons

Enrolment

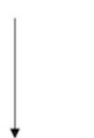

Same day

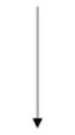

1 week elapsed

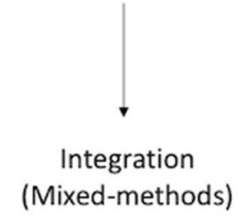

(Mixed-methods)

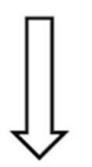

Questionnaires

(Quantitative)

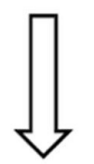

Interviews (Qualitative)

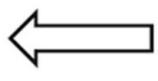

Figure 1 Study timeline and mixed-methods design. Postenrolment day 0: immediately after study enrolment on same day as initial consultation discussing major head and neck surgery.

Studies in Epidemiology and Standard for Reporting Qualitative Research guidelines, ${ }^{11}$ as well as with consideration of best practices in mixed-methods health services research. ${ }^{12-14}$ It is expected that the study period will occur from January 2020 to December 2021.

\section{Mixed-methods design}

The study employs a convergent parallel mixed-methods design whereby quantitative and qualitative portions of the study will occur simultaneously in patients and surgeons (figure 1). The quantitative portion of the study uses patient questionnaires and review of medical records, while the qualitative portion consists of paired semistructured interviews completed on the same patients. Demographic information will be collected, and questionnaires will be administered, at the time of recruitment into the study. Interviews will be conducted 1-week following recruitment into the study. Surgeons will also complete key questionnaires and take part in semistructured interviews.

Utilisation of a mixed-methods design allows for a better understanding of the perceptions of patients and surgeons of their involvement in the complex shared decision-making process. ${ }^{15}$ It also allows for additional questions to be answered, such as the role of decisional self-efficacy and decisional conflict. Additionally, this design allows for gathering information relevant to the development of a decision aid. Use of both quantitative and qualitative methodologies allows a more nuanced approach with generation of richer data. ${ }^{16}$ Qualitative methods are important for eliciting patients' personal

values and beliefs, which are inherent to patient-centred care and the shared decision-making process. The inclusion of semistructured interviews will therefore offer a novel perspective in the shared decision-making process during the oral cancer journey, lending support to the quantitative results.

Integration of the methods will occur when both quantitative and qualitative portions of the study have concluded. Triangulation will be used to assess for metathemes within the data. We will compare patient and surgeon responses during interviews to their quantitative perception of involvement in the shared decision-making process. Integration will be performed by the principal investigator (DG), primary subinvestigator (DF) and experts in mixed-method studies (VER and accompanying team). Decisional self-efficacy and conflict will be compared with themes arising from patient interviews. Triangulation connects data collected from different sources and methods, potentially identifying meaningful information that may have been missed or undiscovered with only one approach. Comparing the data can be a difficult task, particularly when different methodological approaches lead to conflicting outcomes. Triangulation matrix structures will be developed to promote visualisation of the data, with rows representative of findings of shared decision making, decisional conflict, decisional self-efficacy and overall feeling of need for a decision aid, and columns by collection methods. Each row and column of the matrix will be populated and reviewed separately to identify points of convergence and conflict.

\section{Objectives}

The objectives of the study are therefore to:

1. Determine whether patients and surgeons feel a decision aid would be of benefit in the process of decision making.

2. Determine possible information for inclusion in a decision aid.

Corresponding key hypothesis are therefore:

1. Patients with advanced oral cancer requiring resection and reconstruction do not feel optimally integrated in the shared decision-making process.

2. Patients have a low level of decisional self-efficacy and substantial decisional conflict.

3. Surgeons will identify formidable barriers to the use of decision support tools in daily practice.

As this is the first study to attempt to identify the need for a decision aid in major oral cancer surgery, hypotheses 1 and 2 are derived from clinical experience. It is hypothesised that patients and surgeons will believe a decision aid will improve hypotheses 1 and 2, thus substantiating the need for a decision aid.

\section{Population}

This study is multi-institutional including the University Health Network (Toronto, Ontario) and the Queen Elizabeth II Health Science Centre (Halifax, Nova Scotia). This study will enrol patient-physician dyads. As such, 
surgeons may participate in questionnaires multiple times, according to recruitment of their patients, but will participate in interviews on a single occasion. Participants will be recruited by a research assistant or the primary subinvestigator (DF).

Adult ( $>50$ years old) patients with oral cavity cancer advised to undergo surgical ablation and regional or free flap reconstruction will be included in the study. The requirement for major surgery (reconstruction with free or pedicled flaps) was chosen as this patient population represents one with markedly different functional and oncological outcomes compared with patients with less advanced disease. The decision-making process, where the competing risk of death and potential negative functional outcomes must be considered, is thus expectedly different for these patient populations, and must be considered separately in a future study. Patients will still be included if they elect to not undergo surgery. Patients will be recruited in both the preoperative and postoperative periods in order to explore differences between the groups. Postoperative patients needn't have participated in the study during their preoperative phase. Attending surgeons will be eligible if they participate in major oral cavity resection and/or reconstruction, practice at an academic, tertiary care centre and completed a head and neck surgical oncology fellowship. At participating institutions, both the surgical ablations and reconstructions of subsequent surgical defects are completed by head and neck oncological and reconstructive surgeons, all of whom are otolaryngologists.

Patients will still be eligible for participation if they have difficulty communicating. If the patient is unable to communicate during a phone interview, the patient will be presented with the option of being interviewed in person. For patients who require an interpreter, the same principle will apply.

Patient exclusion criteria stipulate:

1. Patients not requiring regional or free flap reconstruction of the primary defect.

2. Patients with T4b and/or M1 disease at time of presentation, or otherwise unresectable disease.

3. Medical comorbidities obviating surgery with curative intent.

4. Previous head and neck cancer requiring surgical ablation and regional or free flap reconstruction.

Surgeon exclusion criteria include:

1. Principal investigator (DG) and site investigator (MC).

\section{Quantitative component}

Quantitative analysis will include both demographic information as well as a series of questionnaires. Demographic information to be collected includes age, medical comorbidities (operationalised as the Charlson Comorbidity Index), history of previous head and neck cancer, education attainment level, history of previous oral cavity resection and/or reconstruction, self-identified communication issues, history of other surgery and history of being involved in the consent process of a family member or spouse. This information will be collected from a distributed form (online supplemental appendix) as well as the patient's electronic chart. Further patient personal health information to be collected includes cancer subsite and proposed reconstructive technique. Surgeon demographic information will be collected, including number of years in practice, residency and fellowship training programmes, and salary structure.

Patients will complete three self-administered questionnaires (The Nine-Item Shared Decision-Making questionnaire (SDM-Q-9), the Ottawa Decision Self-Efficacy scale, and the Decisional Conflict Scale; details below) assessing their perception of involvement in the shared decisionmaking process, perception of decisional self-efficacy and perceived conflict with the decision-making process. Decision aids aim to improve shared decision making, and thus, may reduce conflict and improve decisional self-efficacy. Whether these factors are optimised at baseline will therefore be partially assessed with these questionnaires and integrated with participant themes from the qualitative component of the study. Surgeons will complete the physician equivalent of the questionnaire assessing perceived involvement in the shared decisionmaking process.

\section{Shared decision making}

Multiple different scales for assessment of shared decision making are currently available. As shared decision making is difficult to define, many tools and instruments have been created, with over 40 described in the literature. ${ }^{17}$

SDM-Q-9) is a well-established and validated instrument that measures both the patient's perspective (SDMQ-9) and the surgeon's perspective (SDM-Q-Doc) in the shared decision-making process. ${ }^{18} 19$ It is based on shared decision making being an interactive process in which both parties are equally and actively involved, and share information in order to reach an agreement for which they are jointly responsible..$^{20}$ The scales have been validated in oncological research settings, and each consists of nine items rated on a six-point scale from 'completely disagree' (scored 0 points) to 'completely agree' (scored 5 points). The raw score is transformed, resulting in a range from 0 to 100 . On this scale, 0 represents the lowest possible perception of involvement in the shared decision-making process and 100 represents the highest perception. $^{20}$

\section{Self-efficacy}

The Ottawa Decision Self-Efficacy Scale allows patients to reflect on how confident they feel in making an informed choice about their medical care. ${ }^{21}$ Self-efficacy is the selfconfidence or belief a patient has in their ability to obtain and act on decision-making information, and the support of self-efficacy is essential for patient involvement in the shared decision-making process. ${ }^{22}$ The Ottawa Decision Self-Efficacy Scale is an 11-item questionnaire that has 
been validated in multiple populations and accurately measures patient self-efficacy. ${ }^{22-24}$

\section{Decisional conflict}

Decisional conflict is a state of uncertainty that can arise when a patient is confronted with decisions that have high-risk outcomes or uncertainty around the outcomes. The Decisional Conflict Scale is a 16-item questionnaire with five response categories from 'strongly agree' (scored 1 point) to 'strongly disagree' (scored 4 points). The questionnaire items are separated into information, value clarity, support, uncertainty and effective decision subscales. Transformed final scores above 25 are considered to be clinically relevant conflict. ${ }^{25}$

\section{Qualitative component}

Semistructured, individual interviews have been chosen for their ability to evoke personal experiences and perspectives, especially on sensitive topics such as involvement in the shared decision-making process for cancer. ${ }^{26}$ Interviews will take place on the telephone and be recorded. Patient interviews will be conducted by the primary subinvestigator (DF) under supervision from an experienced qualitative researcher (VER). In order to avoid conflict of interest, surgeon interviews will be conducted by the team (to be recruited) of an experienced qualitative researcher (VER). Patient interviews will take place 1 week after their surgery consultation when the procedure is originally described or 1 week after their first postoperative follow-up. Surgeon interviews will take place at a convenient time for the surgeon and will not be temporally linked to any specific consultation. As noted above, some observations may require in-person interviews for communication requirements. Interviews will be recorded and transcribed verbatim.

The semistructured interview (online supplemental appendix) will consist of questions intended to illicit patient and surgeon experiences in the shared decisionmaking process and their thoughts on the utility of a decision aid. The proposed patient question script will be pilot tested on 2-4 patients to ensure it facilitates discussion on the intended subjects. ${ }^{27}$ The proposed surgeon question script will be pilot tested on 2-4 resident physicians to ensure it facilitates discussion on the intended topics. ${ }^{27}$ Resident physicians will be used as to not diminish the pool of attending surgeons who may be interviewed with the finalised script.

\section{Recruitment}

Potential patients presenting to the head and neck surgical oncology clinic will be identified by healthcare professionals within the circle of care of the patient, including nurses, administrative assistants, attending surgeons and resident physicians. All patients will undergo a noncoercive informed consent process in keeping with Good Clinical Practice Guidelines. ${ }^{28}$

Patients will be recruited for qualitative analysis until theory saturation has been reached, which is estimated to be around 30 patients. ${ }^{29}$ Theory saturation will be defined as no new themes identified from three consecutive interviews. There are 10 head and neck surgeons available and recruited for this study, split between the two participating sites. One surgeon from each site is the respective principal investigator and site investigator of the study and will not be recruited for interview purposes. There will be no remuneration provided for participation in this study.

Additional patients and the same surgeons will be recruited for quantitative analysis alone in order to reach a sample size of 17 observations per group (patient or surgeon) if a sufficient number are not recruited when theory saturation is reached. As this is the first study to assess the need for a decision aid in oral cancer, and the first study to use the SDM-Q-9 and SDM-Q-Doc in this setting, there are no previous studies to draw on for parameters needed for quantitative sample size calculations. For quantitative analysis specifically, we will explore the difference in perceived involvement between patients and surgeons in the shared decision-making process as measured by SDM-Q-9 and SDM-Q-Doc scores. There is no previously defined minimum important difference in the literature, and we have therefore chosen $1 \mathrm{SD}$ to be representative. Other studies have demonstrated expected SD for SDM-Q-9/SDM-Q-Doc score differences of 15 points. ${ }^{30}$ Therefore, a sample of 34 observations (17 per group) would provide a two-sample two-sided t-test (assuming normally distributed data) with $80 \%$ power at alpha $=0.05$ to detect a difference between the two groups of 15 points, assuming an SD of 15 points. As drop-out for the primary outcome is negligible, no additional participants must be recruited. Sample size calculations were performed using SAS University Edition V.9.4 (SAS Institute).

\section{Purposive sampling}

As above, patients will be enrolled until theory saturation is reached, defined as no generation of new themes across research sites. ${ }^{29}$ Purposive sampling will be employed as per standard practices in qualitative research. ${ }^{31}$ Specifically, maximum variation purposive sampling will be used in order to ensure important shared patterns across cases are included and that themes identified have emerged from a purposefully heterogeneous sample representative of the oral cavity cancer population. In order to achieve maximum variation, participants must include both male and female sex, older and younger patients (above and below 70 years of age), those who proceed with surgery and those who do not, patients with both no/ mild comorbidities and those with severe comorbidities, patients cared for by each participating surgeon, patients from both high and low socioeconomic statuses, patients across a spectrum of educational attainment, and patients from various oral cavity subsites requiring differing extent of resection. Each type of patient need not be in a 1:1 ratio, but instead will be representative of the overarching head and neck cancer population. ${ }^{2}$ Patients may 
Table 1 Objectives and corresponding data collection methods

\begin{tabular}{|c|c|c|}
\hline \multirow[b]{2}{*}{ Objective } & \multicolumn{2}{|c|}{ Data collection method } \\
\hline & Quantitative & Qualitative \\
\hline $\begin{array}{l}\text { Determine the perceptions of the patients and surgeons in their } \\
\text { involvement in the shared decision-making process }\end{array}$ & $\begin{array}{l}\text { SDM-Q-9 } \\
\text { SDM-Q-Doc }\end{array}$ & $\begin{array}{l}\text { Multiple semistructured interview } \\
\text { questions, open and closed questions }\end{array}$ \\
\hline Explore the level of decisional self-efficacy of the patients & ODSE & $\begin{array}{l}\text { Semistructured interview questions, open } \\
\text { questions }\end{array}$ \\
\hline Explore the level of decisional conflict experienced by patients & DCS & $\begin{array}{l}\text { Semistructured interview questions, open } \\
\text { questions }\end{array}$ \\
\hline $\begin{array}{l}\text { Determine the current thoughts and viewpoints of patients and } \\
\text { surgeons in regard to the decision-making process for the surgical } \\
\text { management of oral cavity cancer }\end{array}$ & - & $\begin{array}{l}\text { Multiple semistructured interview } \\
\text { questions, open and closed questions }\end{array}$ \\
\hline $\begin{array}{l}\text { Determine whether patients and surgeons feel a decision aid would } \\
\text { be of benefit in this process }\end{array}$ & - & $\begin{array}{l}\text { Multiple semistructured interview } \\
\text { questions, open questions }\end{array}$ \\
\hline
\end{tabular}

DCS, Decision Conflict Scale; ODSE, Ottawa Decision Self-Efficacy Scale; SDM-Q-9, Nine-item Shared Decision-Making Questionnaire; SDM-Q-Doc, Nine-Item Shared Decision-Making Questionnaire, Physician Version.

satisfy multiple factors simultaneously. Targeted purposive sampling will be used, in that only patients fulfilling purposive sampling criteria will be recruited.

\section{Quantitative analysis}

Analysis of the quantitative component of the study will be through descriptive and inferential statistics (table 1). Questionnaire scores will be summarised as means and $\mathrm{SD}$, or medians and IQR, as per the results of tests of normality including the Shapiro-Wilk test, and visual inspection of histograms and $\mathrm{Q}-\mathrm{Q}$ plots. The patient perception of SDM (SDM-Q-9) will be compared with the surgeon's perception (SDM-Q-Doc) using the MannWhitney U-Test or Student's t-test. Differences in surgeon and patient perceptions of shared decision making on quantitative analysis may differ from their perception elucidated in qualitative interviews and may be a challenge for interpretation. However, multiple aspects of the decision-making process are captured, and triangulation will aid in interpreting results as a whole. Patient perceptions of shared decision making (SDM-Q-9) will be correlated with their decisional conflict and decisional self-efficacy (Ottawa Self-Efficacy scale) separately via Pearson correlation or Spearman rank-order correlation.

\section{Qualitative analysis}

Semistructured interviews will be used to elucidate additional information beyond the quantitative analysis (table 1). Thematic analysis of qualitative data using the Braun and Clarke framework will be ongoing throughout the study as an iterative and recursive process. ${ }^{32}$ The general approach to the qualitative data analysis will be to find relevant text within transcripts transcribed verbatim from audio recordings, identify repeating ideas within the relevant text, organise repeating ideas into themes, and finally into an overarching theoretical narrative. ${ }^{33}$ Qualitative analysis will be performed by two experienced qualitative research coders (to be recruited) and overseen by a mixed-methods research expert in order to promote rigour in the analysis (VER). Codebook structure, including codes, themes and the overarching theoretical narrative will be discussed between the trained coders and the primary and principal investigators with content expertise (DF, MC, DG) in order to establish robust, informed results. Theoretical narratives, supporting codes and themes, and direct quotes will be reported.

\section{Patient and public involvement}

No patients were involved in the design of this study.

\section{ETHICS AND DISSEMINATION}

This study has been approved by the research ethics boards of the Nova Scotia Health Authority (Halifax, Nova Scotia, REB 1025068) and the University Health Network (Toronto, Ontario, REB 19-5920).

Personal health information, questionnaire results and interview transcripts will be coded with unique identifiers in order to protect participant identity and privacy. Results dissemination will be in aggregate form only for the quantitative analysis. Qualitative results will include specific quotations, without the patient name or initials, when lending support to higher themes. Due to the nature of the study and population of interest, patients may experience distress when answering questionnaires or participating in the interviews. Distress protocols have been put in place to allow participants to stop involvement at any time, to debrief about their distress, and to escalate care as concern is needed (eg, contacting anonymous mental health reporting phone lines). Such distress may limit study participation and may be an inherent bias introduced to the study. That is, patients that are particularly 
distressed by their treatment may not wish to participate in the study. To reduce this bias, participants will be given the option to participate at various time points, including delayed participation.

Dissemination will be planned through several outlets. Scientific conferences will be targeted for presentation of results to clinicians. Manuscripts will be prepared for peer-reviewed journal publication. Visual abstracts will be prepared for dissemination over social media as appropriate, reaching both clinicians and patients. Results will be presented at institutional and regional meetings. Workshops focusing on shared decision-making in head and neck surgery will be held following future phases of the overarching study. We will also create a head and neck cancer shared decision-making website, with both clinician and patient-oriented sections. Finally, we will distribute results and future decision support tools to patient advocacy groups, and provide brochures and posters to head and neck cancer treatment groups for posting within clinical environments.

\section{Author affiliations}

${ }^{1}$ Otolaryngology -- Head \& Neck Surgery, Queen Elizabeth II Health Sciences Centre and Dalhousie University, Halifax, Nova Scotia, Canada

${ }^{2}$ Institute of Health Policy, Management and Evaluation, University of Toronto, Toronto, Ontario, Canada

${ }^{3}$ Otolaryngology -- Head \& Neck Surgery, IWK Health Centre, Halifax, Nova Scotia, Canada

${ }^{4}$ Toronto Health Economics and Technology Assessment (THETA) Collaborative and Toronto General Hospital Research Institute (TGHRI), University Health Network, Toronto, Ontario, Canada

${ }^{5}$ Krembil Research Institute, University Health Network, Toronto, Ontario, Canada ${ }^{6}$ Otolaryngology -- Head \& Neck Surgery, University of Michigan, Ann Arbor, Michigan, USA

${ }^{7}$ Otolaryngology -- Head \& Neck Surgery, University Health Network, Toronto, Ontario, Canada

${ }^{8}$ Endocrinology, University Health Network, Toronto, Ontario, Canada

${ }^{9}$ Radiation Oncology, University Health Network, Toronto, Ontario, Canada

\section{Twitter David Forner @forner_david}

Collaborators The authors have no competing financial interests. Intellectual disclaimers include: $\mathrm{PH}, \mathrm{DF}$, and AGS perform research in shared decision-making in otolaryngology - head \& neck surgery. JdA, DG, MC, JCI, RG, JRT, SMT, MHR, PJG, DHB, and AGS are practicing head and neck surgical oncologists and treat patients with advanced oral cavity cancer. DF is a resident physician involved in the care of patients undergoing major head and neck surgery. RM is a speech-language pathologist specialising in the treatment of dysphagia in head and neck cancer patients. AMS is an endocrinologist with previous experience in shared decisionmaking and decision aid development for thyroid nodules. VER is a scientist and conducts mixed-methods studies in health technology assessment and health services research. DG conducts research promoting outcomes in elderly patients undergoing major head and neck surgery.

Contributors DF, MC and DG planned the study, developed the initial protocol, and revised protocol versions. PH, AS and AMS offered expert advice on shared decision making and protocol design. VER, JdA and RM offered expert advice on qualitative research and mixed-method study design. PH and VER offered expert advice on needs assessment protocol development. JCI, RG, JRT, SMT, MHR, PG, DB and DHB offered expert advice on the surgical management of oral cancer. RM, JdA and JR offered expert advice on quality of life research. All authors read and approved the final protocol and corresponding manuscript.

Funding The authors have not declared a specific grant for this research from any funding agency in the public, commercial or not-for-profit sectors.

Competing interests None declared.
Patient and public involvement statement Patient and public involvement in the design of this study was not elicited. Subsequent phases in the overarching study may include key patient and public stakeholders.

Patient consent for publication Not required.

Provenance and peer review Not commissioned; externally peer reviewed.

Open access This is an open access article distributed in accordance with the Creative Commons Attribution Non Commercial (CC BY-NC 4.0) license, which permits others to distribute, remix, adapt, build upon this work non-commercially, and license their derivative works on different terms, provided the original work is properly cited, appropriate credit is given, any changes made indicated, and the use is non-commercial. See: http://creativecommons.org/licenses/by-nc/4.0/.

ORCID iD

David Forner http://orcid.org/0000-0002-1014-3545

\section{REFERENCES}

1 Ganzer H, Touger-Decker R, Byham-Gray L, et al. The eating experience after treatment for head and neck cancer: a review of the literature. Oral Oncol 2015;51:634-42.

2 Mifsud M, Eskander A, Irish J, et al. Evolving trends in head and neck cancer epidemiology: Ontario, Canada 1993-2010. Head Neck 2017;39:1770-8.

3 Grobbelaar EJ, Owen S, Torrance AD, et al. Nutritional challenges in head and neck cancer. Clin Otolaryngol Allied Sci 2004;29:307-13.

4 Ziegler L, Newell R, Stafford N, et al. A literature review of head and neck cancer patients information needs, experiences and views regarding decision-making. Eur J Cancer Care 2004;13:119-26.

5 Shay LA, Lafata JE. Where is the evidence? A systematic review of shared decision making and patient outcomes. Med Decis Making 2015;35:114-31.

6 Windon MJ, D'Souza G, Faraji F, et al. Priorities, concerns, and regret among patients with head and neck cancer. Cancer 2019;125:1281-9.

7 Gill SS, Frew J, Fry A, et al. Priorities for the head and neck cancer patient, their companion and members of the multidisciplinary team and decision regret. Clin Oncol 2011;23:518-24.

8 Thomas CM, Sklar MC, Su J, et al. Evaluation of older age and frailty as factors associated with depression and postoperative decision regret in patients undergoing major head and neck surgery. JAMA Otolaryngol Head Neck Surg 2019;145:1170.

9 Shuman AG, Larkin K, Thomas D, et al. Patient reflections on decision making for laryngeal cancer treatment. Otolaryngol Head Neck Surg 2017;156:299-304.

10 McAlpine K, Lewis KB, Trevena LJ, et al. What is the effectiveness of patient decision AIDS for cancer-related decisions? A systematic review subanalysis. JCO Clin Cancer Inform 2018;2:1-13.

11 von Elm E, Altman DG, Egger M, et al. The strengthening the reporting of observational studies in epidemiology (STROBE) statement: guidelines for reporting observational studies. Ann Intern Med 2007;147:573-7.

$12 \mathrm{NIH}$. Best practices for mixed methods research in the health sciences. 2nd ed. Bethesda: National Institutes of Health, 2018.

13 O'Cathain A, Murphy E, Nicholl J. The quality of mixed methods studies in health services research. $J$ Health Serv Res Policy 2008;13:92-8.

14 O'Brien BC, Harris IB, Beckman TJ, et al. Standards for reporting qualitative research: a synthesis of recommendations. Acad Med 2014;89:1245-51.

15 Wisdom J, Creswell J, AHRQ. Mixed methods: integrating quantitative and qualitative data collection and analysis while studying patient-centered medical home models 2013.

16 Creswell JW. Educational research: planning, conducting, and evaluating quantitative. NJ: Prentice Hall Upper Saddle River, 2002.

17 Scholl I, Loon MK-van, Sepucha K, et al. Measurement of shared decision making - a review of instruments. Zeitschrift für Evidenz, Fortbildung und Qualität im Gesundheitswesen 2011;105:313-24.

18 Nejati B, Lin C-C, Imani V, et al. Validating patient and physician versions of the shared decision making questionnaire in oncology setting. Health Promot Perspect 2019;9:105-14.

19 Calderon C, Jiménez-Fonseca P, Ferrando PJ, et al. Psychometric properties of the shared decision-making questionnaire (SDM-Q-9) in oncology practice. Int J Clin Health Psychol 2018;18:143-51.

20 Kriston L, Scholl I, Hölzel L, et al. The 9-item shared decision making questionnaire (SDM-Q-9). development and psychometric properties in a primary care sample. Patient Educ Couns 2010;80:94-9. 
21 O'Connor A. User manual-decision self-efficacy scale. Ottawa: Ottawa Hospital Research Institute 1995.

22 Miller SM, Hudson SV, Egleston BL, et al. The relationships among knowledge, self-efficacy, preparedness, decisional conflict, and decisions to participate in a cancer clinical trial. Psychooncology 2013;22:481-9.

23 Cranney A, O'Connor AM, Jacobsen MJ, et al. Development and pilot testing of a decision aid for postmenopausal women with osteoporosis. Patient Educ Couns 2002;47:245-55.

24 Bunn $\mathrm{H}, \mathrm{O}$ 'Connor A. Validation of client decision-making instruments in the context of psychiatry. Can J Nurs Res 1996;28:13-27.

25 O'Connor A. User Manual-Decisional Conflict Scale (16 item statement format)[document on the Internet]. (c) 1993 [updated 2010]. Ottawa: Ottawa Hospital Research Institute, 2016: 16.

26 Giacomini MK, Cook DJ. Users' guides to the medical literature: XXIII. Qualitative research in health care A. Are the results of the study valid? Evidence-based medicine Working group. JAMA 2000;284:357-62.
27 Dikko M. Establishing construct validity and reliability: pilot testing of a qualitative interview for research in Takaful (Islamic insurance). The Qualitative Report 2016;21:521-8.

28 Dixon JR. The International Conference on harmonization good clinical practice guideline. Quality Assurance 1999;6:65-74.

29 Hagaman AK, Wutich A. How many interviews are enough to identify metathemes in multisited and cross-cultural research? Another perspective on Guest, Bunce, and Johnson's (2006) landmark study. Field Methods 2017;29:23-41.

30 Santema TB, Stubenrouch FE, Koelemay MJW, et al. Shared decision making in vascular surgery: an exploratory study. Eur J Vasc Endovasc Surg 2016;51:587-93.

31 Palinkas LA, Horwitz SM, Green CA, et al. Purposeful sampling for qualitative data collection and analysis in mixed method implementation research. Adm Policy Ment Health 2015;42:533-44.

32 Braun V, Clarke V. Using thematic analysis in psychology. Qual Res Psychol 2006;3:77-101.

33 Maguire M, Delahunt B. Doing a thematic analysis: a practical, stepby-step guide for learning and teaching scholars. AISHE J 2017;9. 